\title{
Review of: "Banana stem and leaf biochar as an effective adsorbent for cadmium and lead in aqueous solution"
}

Hamid Khoshdast

Potential competing interests: The author(s) declared that no potential competing interests exist.

The paper is well written. However, the following points could be improved before publication:

1. The structure is vague. A more organized was better.

2. There is no statistical evaluation on data to confirm the reproducibility. A simple ANOVA would be beneficial. 